\title{
Pressing a Button You Cannot See: Evaluating Visual Designs to Assist Persons with Low Vision through Augmented Reality
}

\author{
Florian Lang \\ florian.lang@ifi.lmu.de \\ LMU Munich \\ Munich, Germany
}

\author{
Tonja Machulla \\ tonja.machulla@tu-dortmund.de \\ TU Dortmund \\ Dortmund, Germany
}
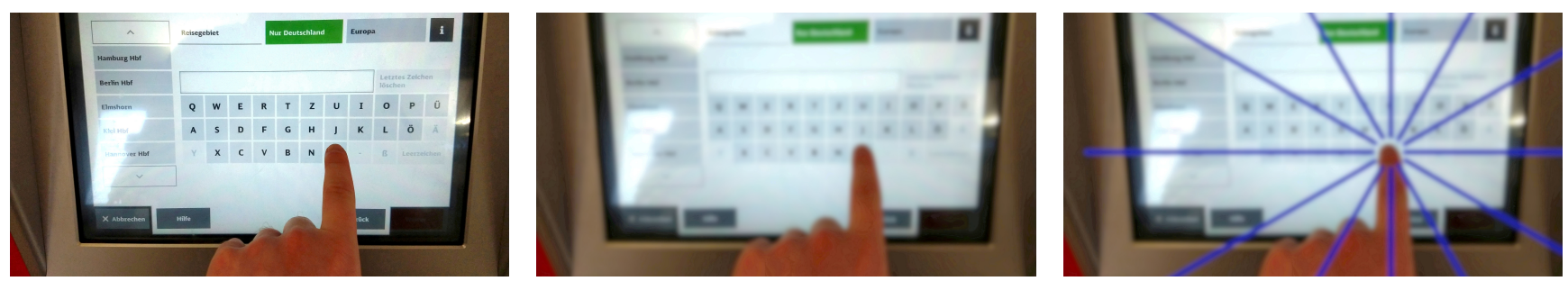

Figure 1: A person without visual impairment can easily complete tasks that require high visual acuity, such as reading and interacting with small interface elements (left). In contrast, a person with visual impairment may have to guess the position of interface elements (middle). To illustrate, we simulated low visual acuity by blurring the image. In our work, we design augmented reality overlays to improve the users' interaction with the interface (right). The augmentation-here in form of radially arranged guiding lines-supports users in finding and precisely activating an interface element.

\begin{abstract}
Partial vision loss occurs in several medical conditions and affects persons of all ages. It compromises many daily activities, such as reading, cutting vegetables, or identifying and accurately pressing buttons, e.g., on ticket machines or ATMs. Touchscreen interfaces pose a particular challenge because they lack haptic feedback from interface elements and often require people with impaired vision to rely on others for help. We propose a smartglasses-based solution to utilize the user's residual vision. Together with visually-impaired individuals, we designed assistive augmentations for touchscreen interfaces and evaluated their suitability to guide attention towards interface elements and to increase the accuracy of manual inputs. We show that augmentations improve interaction performance and decrease cognitive load, particularly for unfamiliar interface layouts.
\end{abstract}

\section{CCS CONCEPTS}

- Human-centered computing $\rightarrow$ Human computer interaction (HCI); Accessibility; Accessibility technologies; Accessibility systems and tools.

\section{KEYWORDS}

Accessibility, Augmented Reality, Low Vision

\section{cc) (1) (ㅇ)}

This work is licensed under a Creative Commons

Attribution-NonCommercial-ShareAlike International 4.0 License.

VRST '21, December 8-10, 2021, Osaka, Japan

(C) 2021 Copyright held by the owner/author(s)

ACM ISBN 978-1-4503-9092-7/21/12.

https://doi.org/10.1145/3489849.3489873

\section{ACM Reference Format:}

Florian Lang and Tonja Machulla. 2021. Pressing a Button You Cannot See: Evaluating Visual Designs to Assist Persons with Low Vision through Augmented Reality. In 27th ACM Symposium on Virtual Reality Software and Technology (VRST '21), December 8-10, 2021, Osaka, Japan. ACM, New York, NY, USA, 10 pages. https://doi.org/10.1145/3489849.3489873

\section{INTRODUCTION}

An estimated number of 441 million people worldwide have a visual impairment (VI) that cannot be corrected by optical lenses. Most affected individuals, even those classified as "legally blind", have some degree of residual visual function and rely on vision in their daily life. In spite of this, novel assistive technology often provides information to other sensory channels such as audition and touch (e.g., $[6,23,24])$. Digital aids that directly support vision exist but have not been innovated much over the last decades; they mainly focus on image enlargement and enhancement. A promising new approach is the visual augmentation of the environment via smart glasses. It has been used for assistance during shopping [35], the climbing of stairs [33], and sign reading [11]. Here, we investigate whether visual augmentation can facilitate the interaction with touchscreen interfaces for persons with VI.

Consider the following vignette: Martha, a woman with VI, is on a business trip in a foreign country and wants to buy a ticket for public transport. Although she can localize the ticket machine and its touchscreen interface, she has difficulty operating the device as her visual acuity is too low to perceive the small and low-contrast interface elements. She recently acquired a pair of augmented reality glasses, which she uses as an aid in many situations. At the ticket machine she uses voice control to convey her intention to the glasses, which then scan a QR code on the machine to load an app that detects and augments the interface to indicate which buttons to press in which 
sequence and also monitors Martha's inputs. Martha can buy her ticket with ease and does not require help from others (see Figure 1).

Touchscreens are becoming increasingly ubiquitous in home appliances and public settings. Just as for Martha, encountering such interfaces in everyday life is challenging for persons with VI as there are often no non-visual means of interaction. Compared to interfaces with physical components, there is little or no tactile feedback as to the location and state of interface elements, and if the interface is interactive, the layout of components may change after input. Over the past two decades, various approaches have been proposed to increase the accessibility of touchscreens. Physical overlays $[14,20]$ add tactile information about the position of interface components. As they have to be prepared ahead of an interaction, they are suitable for static layouts of devices used repeatedly, such as microwave ovens. Newer approaches use 3D printing to add tactile feedback to smartphones [31] or home appliances [7]. More recently, computer vision has been applied to the analysis of static and dynamically changing layouts $[3,8]$ and the guiding of manual inputs via auditory feedback [6].

Head-mounted augmented reality (AR) technology offers a novel approach to improving touchscreen accessibility. AR glasses can receive commands through voice or other connected devices, such as smartphones, and provide auditory or visual feedback while leaving the hands free for interaction with the interface. AR glasses also analyze their surroundings and can recognize the 3D structure of the surrounding scene, markers, QR codes, and text written on signs or interfaces. In this paper, we evaluate the potential of AR visualizations to improve users' interaction with touchscreen interfaces. To this end, we created a set of assistive augmentations that implement two different principles of guiding the users' attention (local highlighting vs. screen-spanning augmentations that provide directional information). A future system could receive the users' intention through voice input, locate an interface, recognize its' current state, and identify the button, which needs to be pressed next to achieve the users' goal. The glasses then can place an augmentation in world coordinates right above that button and align the augmentation with the interface surface to facilitate not only search but also manual inputs of the user.

Our design process consisted of several iterations, each including feedback from users with VI. The final set of designs was evaluated in two scenarios: providing inputs on an interface with a familiar layout, namely a touchscreen keyboard, and searching and activating interface components when the layout is unfamiliar. Our results show that augmentations not only improve the accessibility of touchscreens in terms of speed and accuracy of inputs but also provide a unique solution for a subset of individuals who, without assistive AR, were not able to interact with the touchscreens at all.

\section{BACKGROUND \& RELATED WORK}

Our research builds on prior work on increasing touchscreen accessibility and the use of AR to support individuals with visual impairments in their everyday life.

\subsection{Touchscreen Accessibility}

Touchscreen interfaces are typically designed to rely heavily on users' visual sense. Hence, they pose interaction challenges to users with low vision. They often lack haptic feedback about the location and state of interface elements. Simple gestures like a tap can be troublesome, as the users cannot determine the distance between finger and touchscreen or the location of the target element within it [20]. Scott et al. show that persons with VI have difficulties selecting icons on a computer [30]. This can be attenuated by adjusting the graphical user interface, e.g., by increasing the item size or decreasing the number of items displayed simultaneously [29]. On devices owned by the user, such adjustments are possible through built-in assistive functionality, e.g., the magnifying glass in applications like ZoomText [36]. Smartphones and computers typically also offer non-visual support for interaction such as voice control and text-to-speech. Other options to increase the accessibility of touchscreens are the addition of tactile feedback by creating physical overlays $[7,14,20]$ or active haptic feedback devices to accommodate interface changes [12,31].

In contrast, public or non-adjustable interfaces require external hardware such as an optical magnifier or electronic magnifiers like the portable reading aids from Koolertron [17]. The latter provides additional image enhancement options; however, holding such devices at the correct distance over a touchscreen with one hand and pressing the correct button with the other hand is challenging. For this problem, Guo et al. [8] developed a system using computer vision. As the user hovers and moves their finger over the interface, they receive auditory feedback about the interface elements underneath. A similar system with visual feedback using video-seethrough AR is proposed in [16], albeit without evaluation.

\subsection{Visual Augmentation of the Environment}

Head-mounted technology allows for everyday mobile and handsfree use. Commercial solutions for persons with VI exist, providing either auditory or visual assistance (e.g., OrCam [23] and NuEyes 2 [22], respectively). The latter has various advantages: it is better suited for persons with hearing impairments, in noisy environments, or in situations where audible auditory feedback might not be desirable due to privacy concerns. If the user has sufficient residual vision, localization of targets is faster and more accurate than when using auditory feedback. However, visual feedback in the form of magnification, e.g., provided by NuEyes, amplifies perceived motion from head movements, and can lead to motion sickness. Further, crucial information outside of the magnified area might be missed.

In contrast to commercial solutions, which are still fairly simple in their functionality, scientific research often targets more complex problems. González proposed a system based on video-feedthrough and image manipulation for persons with age-related macula degeneration (AMD) in 2011 [19]. It included an option to correct distorted vision (metamorphopsia), which is common in AMD, by modifying the video image through a user-specific inverse transformation. In 2015, Zhao et al. published a similar system implementing image modifications, e.g., contrast enhancement and magnification [34], followed by a more versatile system for the assistance of individuals with VI in virtual reality (VR) in 2019 [32]. In the latter case, additional information about the virtual environment was used to highlight important objects or project the scene layout to the center of the visual field to support users with peripheral vision 
loss. Another solution helps users to navigate and use stairs more easily by highlighting the edges of the steps [33]. Sterns et al. proposed an electronic magnifier using the Microsoft HoloLens and a finger-mounted camera [27], which ports the functionality of an electronic magnifier to an HMD. The finger-mounted camera is not affected by head movements and the desired area can be selected easily. To assist users during indoor navigation, Huang et al. propose an approach to recognize text on signs and display it in large and high-contrast virtual letters to the user [11].

However, AR technology offers possibilities beyond image enhancement and magnification. Information can be provided in different forms and levels of abstraction, adapted to the visual capabilities of the user [18]. Augmentations can guide attention in strenuous tasks like object search for users with full visual acuity by displaying symbols as pointers [26], project the scene into a map [5], guide attention directly towards the object [25], or show arrows at the borders of the screen [1]. Zhao et al. presented AR clues for visual search during shopping to assist users with visual impairment [35] They augmented the desired product by placing an image over it and adding motion, flicker, or highlighting. We extend this research by investigating how AR can assist manual inputs in addition to visual search and by designing augmentations that users with visual impairment can perceive.

\section{DESIGN PROCESS FOR AUGMENTATIONS}

We targeted our design towards people who: i) had difficulties recognizing interactive elements on touchscreen devices in public settings, and ii) reported sufficient residual vision to perceive defined contours of bright light (e.g., perceiving a line of LEDs as a line rather than a diffuse light field). People with a stronger impairment of vision would require an alternative approach such as perceivable flicker coupled to hand motions or auditory support. Our process consisted of four phases: (1) initial design from literature, (2) qualitative evaluation of early prototypes with three persons with VI, (3) a quantitative pilot study with seven persons with VI, and (4) a quantitative study with 16 participants (described in sections 4-6). In the first phase, we developed a large set of augmentations based on related work in $\mathrm{HCI}[25,35]$ and perceptual psychology [4, 21]. The designed augmentations can be divided into two groups: global guides and local highlights. In both variants the center of the augmentation is locked above a relevant interface element, to improve targeting of manual input.

Local highlights are locally presented cues. They draw attention fast and involuntarily to the interface component that is to be activated next. They are small and result in little occlusion or visual clutter. At the same time, they are difficult to find if, during their onset, they are located outside the augmentable area or inside the user's visual field defect. The feedback in phases 2 and 3 suggested that only one of these augmentations was easy to perceive and sufficiently supported targeting.

Global guides are large virtual objects that span the entire interface. They will always be partly visible, regardless of which area of the interface the user is currently looking at. However, attention has to be shifted voluntarily towards the center of the augmentation, which takes comparably more time. To counteract this issue, we created variants with directional information, such as ArrowheadCross, or with moving elements since motion is easily detected in peripheral vision [21] and can be used to direct attention. Directional information also supports the user if the target is located outside of the area that is augmentable by the HoloLens and the user needs to move their head. For the global guides, we constructed crosses of 2-6 lines with equal angles between them. If possible a horizontal and vertical line were included. In phases 2 and 3, we excluded several of these variants as their complexity resulted in occlusion of the interface and perceptual clutter of background and overlay elements. Additionally, some moving augmentations caused motion sickness and were excluded.

The final set of augmentations used in phase 4 is shown in Figure 2. All augmentations have an aperture at the center, as participants in phases 2 and 3 had remarked that they preferred to see the interface element behind the augmentation. Phases 2 and 3 were also used to determine intensity, color, line width and number, total size, rotation angle, and size of the aperture for phase 4. A more in depth description of our design process can be found in the supplemental material.

\section{RESEARCH QUESTIONS}

In this work, we evaluate the potential of AR to support persons with VI when interacting with touchscreen displays. We are interested in whether the user's performance improves with visual feedback and if there are differences between augmentations. This can be expressed in three research questions (RQ1 to RQ3) and corresponding hypotheses regarding the outcome (H1 to H3).

RQ1: Does visual augmentation aid persons with VI in their interactions with interactive touchscreens? Visual augmentations have been used to guide a user's attention towards out-of-view or poorly perceptible objects [25, 35]. In addition, we expect augmentations to assist the alignment of the user's finger with the target. Improvements in both visual search and goal-directed reaching should manifest as faster or more accurate inputs. Speedaccuracy trade-offs should be excluded as potential explanations for changes in performance between conditions. This leads to H1: The performance will improve if the interface is augmented. In particular, we expect improvements in input speed and/or accuracy.

RQ2: Is there a difference between augmentations? We included five augmentations into the evaluation. Most generally, we expect interindividual differences in participants' preferences due to the heterogeneous catalog of symptoms typically displayed by the target group (i.e., persons with "low vision"). The expression of a desire for customizability is a common result in the evaluation of assistive technology [15]. However, based on previous work and participants' feedback during the design process, we also developed some concrete expectations with regard to the augmentations' relative usability across a group of users, which we state below.

RQ2.1: How do global guides compare to local highlights? We compare the local highlighting augmentation against the various forms of global guides. The two types of augmentation support different attentional strategies and also differ in their form factor. For these reasons, we expect results to be dominated by participants' individual perceptual requirements and strategies. We formulate a non-directional hypothesis to test whether one set of factors 


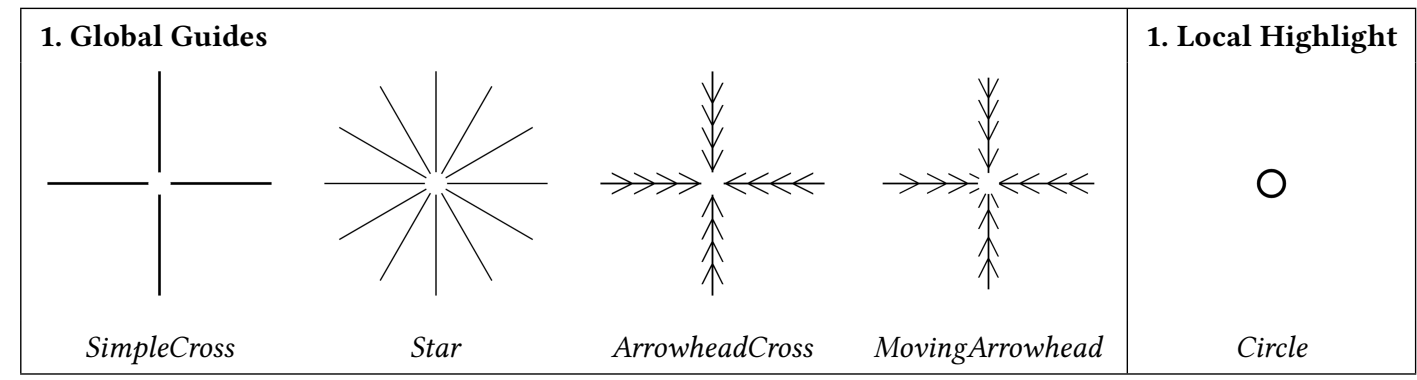

Figure 2: Here, we show all augmentations that were used in the final study.

dominates over the other: H2.1: The performance when using local highlights will differ from using global guides.

RQ2.2: Does adding directional information to a simple cross effectively guide attention towards a target? The augmentations are of varying levels of complexity. In particular, they differ in the amount of directional information they provide. Here, we compare a simple global guide with little directional information against more complex global guides with explicit unidirectional information. We expect the latter to provide a search benefit, specifically if the target lies outside the augmentable area or if visual impairments preclude participants from viewing the augmentation as a whole. We formulate this as H2.2: Global guides providing directional information will lead to better performance compared to a simple global guide.

RQ2.3: Does motion provide an additional directional benefit? Here, we compare two global guides. The first, ArrowheadCross, conveys directional information through form alone. For the second global guide, we animated the augmentation to provide an additional and redundant directional cue through motion. Redundancy in perceptual cues often leads to increases in performance in terms of increases in speeded responses and the accuracy of detection and selection of targets. This leads to H2.3: The performance will improve when motion is added as an additional cue to a static augmentation.

RQ3: Does the provided AR assistance influence the perceived workload of the users? Ideally, assistive technology does not only enable the execution of challenging or impossible tasks but also makes execution (subjectively) easier. Tasks that require high visual acuity are characterized by an information-dense visual environment. They are often perceived as strenuous by persons with VI and are consequently avoided. During the design process, we excluded any augmentation that was reported to be difficult to perceive or interpret, increase visual clutter, or distracted in other ways (e.g., by causing motion sickness). The final set of augmentations provides simplified task-relevant information at a higher contrast, reducing the requirements for the minimal visual acuity necessary to accomplish the task at hand. For these reasons, we assume that AR-assisted interactions with interface components will be judged as less demanding. This leads to H3: The subjective workload will decrease when assistive augmentations are provided.

\section{METHODOLOGY}

We evaluated the augmentations in two scenarios with similar designs. In the first scenario, FamiliarLayout, we augmented a familiar interface to simulate devices the user regularly interacts with or layouts found in similar form across different devices. We chose a standard keyboard layout because it is likely to be familiar to participants and is commonly encountered in public touchscreen interfaces. Further, we evaluated the augmentation of unfamiliar interfaces in a second scenario, UnfamiliarLayout. To retain comparability, we used the keyboard's color scheme and size, but randomized the letters on the buttons to create an interface unknown to the participant. We asked participants not to correct wrong inputs because in the pilot study, users varied strongly in their strategies for correcting inputs. Some had memorized the target's position from the initial interaction and thus acted a lot faster, while others were unsettled and double-checked the letter several times, resulting in much slower performance. Both outcomes do not reflect our measure of interest, namely input speed with augmentation. Ethical approval for this study was obtained from the IRB.

Participants. We recruited 16 participants ( 8 female, 8 male, 0 diverse; mean age of 54.9 years with standard deviation of 18.2 years, details see Table 1). Interested persons were screened over the telephone according to the criteria mentioned in Section 3. Before the study, we measured participants' visual acuity and tested whether they were able to use AR glasses. Three of the 16 participants had difficulties. For them, we switched to a demonstration-like approach, gathering qualitative feedback. As their reasons for not being able to use the technology provide insights into the limitations of our approach, we report them in Section 6.3. Participants were compensated with $12 €$ per hour for 2-3 hours of their time. To increase the accessibility of the study, we also reimbursed travel expenses and offered meeting participants at the nearest subway or bus station.

Technical Setup. The technical setup consisted of a VIEWPixx3D light $(24 \mathrm{inch}, 1920 \times 1080$ resolution at $120 \mathrm{~Hz}$ ) monitor, including a TOUCHPixx [28] add-on (24 inch touchscreen, $4096 \times 4096$ resolution). As AR glasses, we used the Microsoft HoloLens. Monitor, touchscreen, and HoloLens were calibrated with regard to each other for each participant to account for user-dependent viewpoint off-sets. Each participant was seated about $45 \mathrm{~cm}$ from the vertical monitor and free to move their heads to find their optimal view of the UI to reflect real-life setups. Large movements were only observed during baseline input when many drew their faces close to the UI. Still, overall performance was worse in this condition.

Design. We used a within-subject design for each of two scenarios: FamiliarLayout and UnfamiliarLayout. Each consisted of 
Table 1: A detailed list of all participants. If multiple medical conditions were reported, we list the one with the greatest impact on the participant's sight.

\begin{tabular}{ccccl}
\hline ID & Age & Gender & $\begin{array}{c}\text { Visual } \\
\text { Acuity }\end{array}$ & Diagnosis \\
\hline P1 & 82 & $\mathrm{~m}$ & 0.18 & Dry AMD \\
P2 & 81 & $\mathrm{f}$ & 0.17 & AMD \\
P3 & 20 & $\mathrm{~m}$ & 0.02 & Leber congenital amaurosis \\
P4 & 24 & $\mathrm{f}$ & 0.14 & Bilateral iris coloboma \\
P5 & 67 & $\mathrm{~m}$ & 0.04 & Retinal detachment \\
P6 & 66 & $\mathrm{~m}$ & 0.13 & Albinism \\
P7 & 40 & $\mathrm{~m}$ & 0.22 & Retinitis pigmentosa (RP) \\
P8 & 67 & $\mathrm{f}$ & 0.16 & Retinitis pigmentosa \\
P9 & 51 & $\mathrm{f}$ & 0.10 & Macula degeneration \\
P10 & 58 & $\mathrm{f}$ & 0.01 & Glaucoma \\
P11 & 49 & $\mathrm{f}$ & 0.01 & Cataract and glaucoma \\
P12 & 66 & $\mathrm{~m}$ & 0.06 & AMD \\
P13 & 55 & $\mathrm{~m}$ & 0.03 & Macula degeneration \\
P14 & 27 & $\mathrm{f}$ & 0.01 & Nystagmus \\
P15 & 65 & $\mathrm{f}$ & 0.04 & Morbus Stargardt \\
P16 & 61 & $\mathrm{~m}$ & 0.01 & Inverse RP \\
\hline
\end{tabular}

six conditions-the five augmentations plus the baseline condition without AR assistance. The presentation order of the conditions was counterbalanced within each scenario using a balanced Latin square design. In each condition, we requested 24 manual inputs for FamiliarLayout and 25 for UnfamiliarLayout.

Task \& Stimuli. For the FamiliarLayout scenario, we implemented a virtual keyboard, which was similar in size, appearance, and contrast to the keyboard of the ticket machine of the national train company (see Figure 1). Each key was $17 \mathrm{~mm}$ in width and length. The keyboard had auditory feedback, i.e., each input triggered a click sound. We used black text, a grey value of 0.7 for buttons and 0.88 as background. In the augmentation conditions, participants maintained a distance of roughly 0.45 meters from the screen to retain comparability between augmentations. At this distance, the augmentable area of the HoloLens is smaller than any of the global augmentations. Therefore, global guides were only partially visible. During Baseline, participants freely choose the distance at which they viewed the keyboard, as this more closely resembles their natural behavior. This approach ensured that any improvement resulting from the use of augmentations would be based on participants' optimal performance in the Baseline condition. We used the five augmentations Circle, SimpleCross, Star, ArrowheadCross, and MovingArrowheadCross as depicted in Figure 2. We used the color red, an intensity of $40 \%$ and a central circular aperture with a diameter of $17 \mathrm{~mm}$ for all conditions. The line width was $3 \mathrm{~mm}$ for Circle, $1 \mathrm{~mm}$ for Star, and $2 \mathrm{~mm}$ for others and the total size was $23 \mathrm{~mm}$ for Circle and $531 \mathrm{~mm}$ (screen width) for others. In each condition, the task was to enter four six-letter words, which were randomly (nonrepeating) selected from a list of city names.
In the UnfamiliarLayout scenario, the presented layout was identical to the previous scenario but contained only square keys, i.e., no space bar. Overall, there were 33 keys arranged in a $3 \times 11$ grid. The keys were randomly labeled with (repeating) letters from the alphabet. On each trial, labels were re-randomized, one of the letters from the set $\{\mathrm{O}, \mathrm{M}, \mathrm{N}, \mathrm{Q}, \mathrm{D}, \mathrm{C}, \mathrm{G}\}$ was selected as a target, and participants had to find and press all five occurrences of this target within the interface.

Procedure. All participants were seated in front of the touchscreen and acquainted with the HoloLens. Next, we explained the goal of our project and informed participants about their rights regarding their data and that they could abort or pause the experiment at any time. We also collected demographic and medical information, followed by a brief explanation and demonstration of all augmentations in the HoloLens. Participants were instructed to prioritize accuracy over speed. The task was explained before each scenario and there was a training phase before each condition.

For the FamiliarLayout, a word was shown on full screen and read (text-to-speech) to the participant. They were encouraged to ask in case of unclear spelling. The participant then proceeded by entering the word over the keyboard. After six buttons were pressed, the next word was shown and read. For the UnfamiliarLayout, the target letter was displayed first followed by the interface consisting of buttons with randomly positioned letters as described above. When a button was pressed, it became invisible to avoid repeated selection by participants. Five sets were presented.

In both scenarios, participants filled in the NASA-TLX [10] questionnaire after each condition.

Measures. To evaluate the suitability of the different augmentations, we collected three types of data: First, we measured objective performance in the form of task completion times (TCT) and accuracy of button presses in form of the error rate (ER). We analyze TCT at the level of single button presses, i.e., how long it takes to find and press single buttons. The timer measuring the TCT of the first button press was started by the participant: after receiving the task (e.g., enter a specific city name), they tapped the touchscreen once for the interface to appear. After that, we measured the time between successive button presses (excluding times for wrong button presses). Second, we asked for subjective feedback in the form of comments and suggestions after each condition and the nomination of a preferred augmentation after each scenario. Third, we obtained participants' self-evaluation regarding perceived workload through the NASA-TLX questionnaire [10].

\section{RESULTS}

In this section, we present the quantitative results. Participants P10, $\mathrm{P} 11, \mathrm{P} 12$, and $\mathrm{P} 13$ did not provide a dataset in the baseline condition as they were not able to perceive the interface elements. However, the four participants successfully interacted with the touchscreen in the conditions where assistive augmentations were provided. Therefore, their data is included in the statistical analysis of RQ2, while the analysis of RQ1 and RQ3 where we compare Baseline against the other conditions is based on the remaining participants' data. We report the NASA-TLX scores, the task completion time (TCT) in seconds and the error rate (ER), i.e., the number of wrong 
inputs divided by the total number of inputs. A wrong input is either a button press on any interface element other than the target or a press on the touchscreen that misses the entire interface.

It should be noted that the heterogeneity in participants' symptom complexity and residual visual ability results in considerable variability in baseline abilities, as well as in the augmentation that objectively provides optimal support and the augmentation that is subjectively perceived as most helpful. This interindividual variability is not uncommon when assistive technologies are tested [13] and group-level analysis can mask individually helpful solutions, particularly when participants perform differently. Therefore, we supplemented group-level analysis with an in-depth analysis at the individual level, specifically evaluating subjective verbal feedback.

Analysis. Data was analyzed along the three research questions stated in Section 4. We first report descriptive and inferential statistics at the group level. For the analysis of RQ1 and RQ2, we conducted a series of pairwise comparisons in the form of planned contrasts. Each contrast compares successively smaller subsets of the data that entered into the previous contrast, with no subset being used twice. For this reason, the family-wise error rate is not subject to inflation and there is no need to correct for multiple comparisons [2, Ch. 10.4]. The contrasts are: for RQ1, we compare Baseline to the average of all augmented conditions $\{$ Augmented\}, i.e., mean(Star, ArrowheadCross, MovingArrowheadCross, SimpleCross, Circle). For RQ2.1, we compare the local highlight Circle against the global guides \{Cross\}, i.e., mean(Star, ArrowheadCross, MovingArrowheadCross, SimpleCross). For RQ2.2 we examine the difference between simple and complex global guides, comparing SimpleCross against \{Complex cross\}, i.e, mean(Star, ArrowheadCross, MovingArrowheadCross), and for RQ2.3, we compare ArrowheadCross against MovingArrowheadCross.

We conducted pairwise t-tests with Welch correction for each of the scenarios (FamiliarLayout and UnfamiliarLayout), each of the measures (TCT and ER), and each of the four research questions. For RQ1, RQ2.2, and RQ2.3, we stated directional hypotheses and therefore used one-tailed tests. The tests for RQ1 have eight degrees of freedom (nine participants); tests for the other research questions have 12 degrees of freedom (13 participants). All results are summarized in Table 2

For the comparison of NASA-TLX ratings across conditions to answer RQ3, we used Wilcoxon signed-rank tests, because the ratings are not normally distributed. We further provided the data for RQ1 and RQ3 in graphical form in Figure 3. For RQ2 no significant results can be reported.

\subsection{Task Completion Time and Error Rate}

Here, we summarize the results and provide arithmetic means (M) with the associated standard error of the mean (SE). For UnfamiliarLayout, TCT decreases significantly when augmentations are provided during interaction (RQ1; Baseline $\mathrm{M}=5.7 \mathrm{~s}, \mathrm{SE}=3.1 \mathrm{vs}$. $\{$ Augmented $\} \mathrm{M}=3.5, \mathrm{SE}=3.9)$. For FamiliarLayout, this improvement is not significant (RQ1; Baseline $\mathrm{M}=2.7 \mathrm{~s}, \mathrm{SE}=1.9 \mathrm{vs}$. $\{$ Augmented $\mathrm{M}=1.9 \mathrm{~s}, \mathrm{SE}=0.5)$. Further, we find that augmentations reduce ER ((RQ1; FamiliarLayout: Baseline $\mathrm{M}=0.33, \mathrm{SE}=0.20$ vs. $\{$ Augmented $\} \mathrm{M}=0.19, \mathrm{SE}=0.22$ ); UnfamiliarLayout: Baseline $\mathrm{M}=$ $0.24, \mathrm{SE}=0.26$ vs. $\{$ Augmented $\} \mathrm{M}=0.18, \mathrm{SE}=0.23))$; this finding
Table 2: This table displays the result of the 16 t-tests for $R Q 1$ and $R Q 2$. The degrees of freedom are eight for RQ1, 12 otherwise. The cells list $t$ and $p$-values.

\begin{tabular}{|c|c|c|c|c|c|c|c|c|}
\hline & \multicolumn{4}{|c|}{ FamiliarLayout } & \multicolumn{4}{|c|}{ UnfamiliarLayout } \\
\hline & \multicolumn{2}{|c|}{ TCT } & \multicolumn{2}{|c|}{ ER } & \multicolumn{2}{|c|}{ TCT } & \multicolumn{2}{|c|}{ ER } \\
\hline & $\mathrm{t}$ & $\mathrm{p}$ & $\mathrm{t}$ & $\mathrm{p}$ & $\mathrm{t}$ & $\mathrm{p}$ & $\mathrm{t}$ & $\mathrm{p}$ \\
\hline RQ1 & 1.31 & 0.11 & 2.12 & 0.03 & 2.04 & 0.04 & 1.26 & 0.12 \\
\hline RQ2.1 & 2.12 & 0.06 & 1.66 & 0.12 & 1.36 & 0.20 & 1.51 & 0.16 \\
\hline RQ2.2 & 0.13 & 0.45 & -2.20 & 0.98 & -1.86 & 0.96 & -1.25 & 0.88 \\
\hline RQ2.3 & 0.25 & 0.40 & -0.98 & 0.83 & -0.75 & 0.77 & 1.31 & 0.11 \\
\hline
\end{tabular}
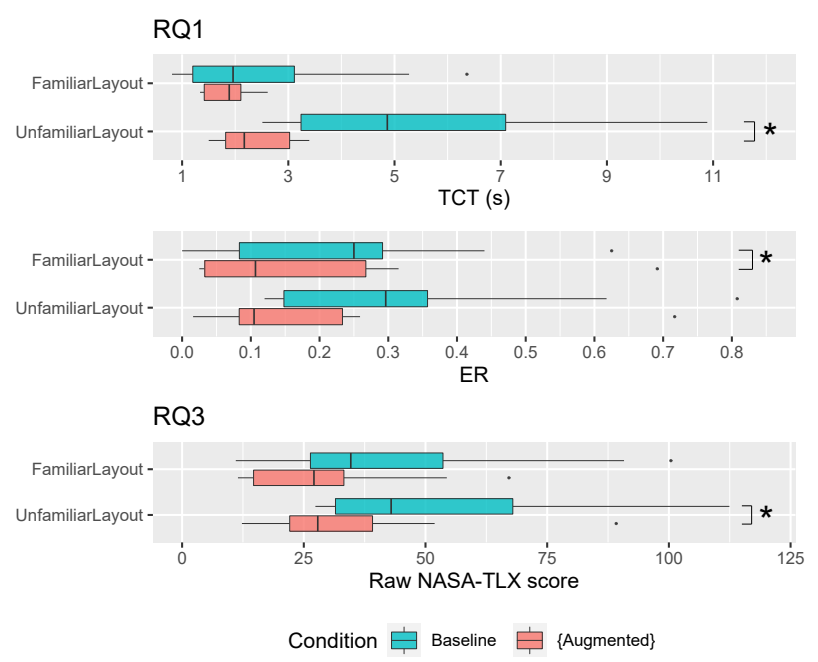

Figure 3: Here, we show TCT (top) and ER (middle) for RQ1 and the Nasa-TLX Scores (bottom) for RQ3. Each of the three graphs shows the results for FamiliarLayout above the data for UnfamiliarLayout. For each pair of results the baseline condition in teal is above the combined data of all augmented conditions in red. Significant results are marked with a star.

is significant for FamiliarLayout. However, to reiterate, there were four additional participants who could not complete the baseline but were able to interact with the interface when an augmentation was provided. Further, we see a large variability in the TCT in the Baseline condition (top graph in Figure 3), even without the participants who could not complete this condition. When the interface is augmented, this variability is numerically smaller.

Regarding RQ2, we did not find evidence that one type of augmentation provides better assistance than another when analysed across participants. All values are given in Table 3.

\subsection{Preferred Visualization and Workload}

Comparing Baseline against \{Augmented\} yields a 33\% lower raw NASA-TLX score in FamiliarLayout and a significantly $41 \%$ lower value for UnfamiliarLayout $(\mathrm{Z}=1.48, \mathrm{p}=0.08$ and $\mathrm{Z}=2.31, \mathrm{p}=0.01$, 
Table 3: This table displays the mean (M) and standard error of the mean (SE) of TCT and ER for research question 2.

\begin{tabular}{|c|c|c|c|c|c|c|c|c|}
\hline & \multicolumn{4}{|c|}{ FamiliarLayout } & \multicolumn{4}{|c|}{ UnfamiliarLayout } \\
\hline & \multicolumn{2}{|c|}{ TCT (s) } & \multicolumn{2}{|c|}{ ER } & \multicolumn{2}{|c|}{ TCT (s) } & \multicolumn{2}{|c|}{ ER } \\
\hline & $M$ & SE & $\mathrm{M}$ & $\mathrm{SE}$ & $\mathrm{M}$ & SE & M & SE \\
\hline RQ2.1 & & & & & & & & \\
\hline$\{$ Cross $\}$ & 2.3 & 0.7 & 0.28 & 0.23 & 3.7 & 3.3 & 0.26 & 0.23 \\
\hline Circle & 1.9 & 0.8 & 0.21 & 0.27 & 3.3 & 2.5 & 0.21 & 0.20 \\
\hline RQ2.2 & & & & & & & & \\
\hline SimpleCross & 2.3 & 0.9 & 0.21 & 0.20 & 3.3 & 2.7 & 0.23 & 0.26 \\
\hline$\{$ Complex cross\} & 2.3 & 0.7 & 0.30 & 0.25 & 3.8 & 3.5 & 0.27 & 0.23 \\
\hline RQ2.3 & & & & & & & & \\
\hline ArrowheadCross & 2.3 & 0.8 & 0.27 & 0.26 & 3.9 & 3.2 & 0.31 & 0.26 \\
\hline MovingArrowhead. & 2.3 & 0.9 & 0.32 & 0.22 & 4.1 & 3.8 & 0.25 & 0.24 \\
\hline
\end{tabular}

respectively). The corresponding data is shown in the bottom graph of Figure 3 (RQ3; FamiliarLayout: Baseline $\mathrm{M}=46, \mathrm{SE}=31$ vs. $\{$ Augmented $\} \mathrm{M}=30, \mathrm{SE}=19$; UnfamiliarLayout: Baseline $\mathrm{M}=55$, $\mathrm{SE}=32$ vs. $\{$ Augmented $\} \mathrm{M}=36, \mathrm{SE}=23)$. We have no evidence that one type of augmentation provides a larger benefit than another across all participants.

In addition to the cumulative score, the subscales of the NASATLX are commonly analyzed individually [9]. We find that providing an augmentation lowers the user's perceived mental demand and effort (FamiliarLayout: $\mathrm{Z}=2.0, \mathrm{p}=0.03 ; \mathrm{Z}=2.0, \mathrm{p}=0.03$, UnfamiliarLayout: $\mathrm{Z}=1.6, \mathrm{p}=0.06 ; \mathrm{Z}=2.3, \mathrm{p}<0.01$ ). If the interface is unfamiliar to the user, the augmentation additionally reduces the perceived temporal $(Z=2.3, p<0.01)$ and physical $(Z=1.7, p<0.05)$ demand.

Individually, stated preferences of augmentation type varied and appeared to depend on the participants' visual abilities and compensation strategies. Yet, there are some clear patterns. No participant chose ArrowheadCross or MovingArrowheadCross as their preferred augmentation; in fact, these were often reported as least favorite. They were described as "too much"; in particular MovingArrowheadCross was described as difficult to perceive. Nevertheless, they were still preferred over no augmentation. Five out of 13 participants preferred Circle, five preferred SimpleCross and three favored Star.

\subsection{Limitations of Assistive Augmentations}

Three participants-P14, P15, and P16-were not able to complete the quantitative part of the study. We consider their feedback important as it provides insights into some of the limitations of the proposed approach in terms of target group and current technological implementation.

P15 has a large central scotoma. She entered 3.5 words with an error rate of 0.42 using SimpleCross and Circle without any training. She stated that recognizing the augmentations was possible but very strenuous since she had to retain a sustained focus of attention in the visual periphery. Accordingly, she reported not interacting with touchscreens if the interface is unknown ("I would never do that.") and evading tasks requiring high visual acuity during everyday life.
P14 focuses on one small area at a time due to her nystagmus. For this reason, she was not able to easily perceive the augmentation as a whole ("I follow the line [of SimpleCross] until the center"). To input characters (with and without augmentation) P14 moved very close (approximately $5 \mathrm{~cm}$ ) to the screen. The small distance proved challenging for the HoloLens as tracking was frequently lost. Nevertheless, she was able to input six words with an error rate of 0.10 . She stated that she would prefer the HoloLens over no help, but her smartphone (as a digital magnifier) over the HoloLens.

P16 also had a large central scotoma covering $15^{\circ}$ of his visual field. The augmentable area (display) of the HoloLens covers $17^{\circ}$ vertically. Therefore, he could not perceive the augmentation when the HoloLens was worn as intended and had difficulties adjusting the device on his head such that the augmentation was visible. Eventually, he used one hand to hold the HoloLens in a suitable position. This allowed correct and precise inputs after the calibration. However, inputs towards the end of each condition were more prone to errors due slightly moving the device resulting in a loss of calibration. As the task was perceived as exhausting, especially holding the HoloLens, the study was aborted. He stated that the current technology "is diametrical to [his] symptoms", i.e., his visual field defect coincided with the augmentable area of the HoloLens, and that "other patients with a loss of peripheral vision or AMD [with a smaller central scotoma] might benefit [from the augmentation]".

To summarize, using our designs to augment a touchscreen might not be suitable for: i) individuals with large central visual field defects, either because peripheral perception requires a substantial voluntary effort or because the augmentable area of current headmounted AR technology is too narrow and cannot be freely placed within the user's field of vision, ii) individuals with impairments of a wholistic perception of screen-spanning augmentations, and iii) individuals who require close-up inspection of the interface.

\section{DISCUSSION}

Overall, our results demonstrate that augmentations can assist persons with VI when interacting with touchscreen interfaces. Our main findings are four-fold: First, augmentations decrease the time required and the number of wrong inputs when users interact with a device. Second, augmentations allow persons that previously could not use a touchscreen device to overcome this obstacle. P9 noted that the glasses facilitated interaction, "I can often not get close enough to touchscreens [in public to perceive their components]. I imagine this is a lot easier with [smart]glasses". Third, augmentations subjectively reduce the effort associated with the interaction with inaccessible touchscreens. And lastly, we find that persons vary considerably regarding which type of augmentation provides a benefit. In the following, we discuss these findings in more detail, integrating the quantitative results from the previous section with qualitative feedback from the participants.

\subsection{Augmentations Lead to Faster and More Accurate Inputs (RQ1)}

Our findings confirm that assistive augmentations help persons with VI to interact with touchscreen devices faster and more accurately. However, closer inspection of the data provides a more 
nuanced picture when considering familiarity with layouts and the participant's visual capabilities.

For familiar layouts, we found that, on average, participants made significantly fewer errors: when an augmentation was present $18 \%$ percent of inputs were wrong compared to $24 \%$ during the baseline condition. Additionally, the time it took to find and activate an interface element decreased, on average, from $2.7 \mathrm{~s}$ in the baseline condition to $1.9 \mathrm{~s}$ when an augmentation was present. This latter difference was not significant, which may be due to the large variability found between participants. Some participants profited immensely from an augmentation, e.g., P1 improved from 6.4s in the baseline condition to $1.4 \mathrm{~s}$ if an augmentation was presented and P7 improved from 5.3s to 2.1s, stating, "I can see the individual buttons, but no letters." However, others, particularly those who could discriminate the letters, were actually hampered by the augmentations. $\mathrm{P} 8$ required $0.8 \mathrm{~s}$ in the baseline condition, but $1.8 \mathrm{~s}$ with the support of an augmentation. She stated, "I see the keyboard. I see the letters, and I can read them because I know them". This opposing trend may have masked significant improvements by people from the first group. Despite these interindividual differences, overall performance was numerically faster and less variable when using augmentations. In sum, the augmentation of a familiar layout provides a benefit to the user if the interface cannot be perceived; otherwise, it may hinder the user.

For unfamiliar layouts, participants' performance improved even more than for familiar layouts. If an augmentation was presented, participants required significantly less time for an input (3.5s vs. $5.7 \mathrm{~s}$ in the baseline condition). At an individual level, the largest improvement was a decrease from 10.9s during the baseline condition to $3.4 \mathrm{~s}$ with an augmentation by P2. Augmenting the interface also improved the accuracy for single participants. P7 showed the largest improvement with $81 \%$ of inputs being wrong during the baseline condition and $14 \%$ when an augmentation was present.

In conclusion, our results show that the majority of persons with VI profit from assistive augmentations. The average benefit is likely enhanced if the users can choose their preferred augmentation, customize it, and decide when to use AR assistance. For familiar layouts, users who can still perceive the interface may perform better without any assistance. However, when the interface cannot be perceived the user benefits from augmenation. For unfamiliar interfaces, the augmentation not only assists in correctly hitting the interface element but also in finding it. Here, the results show that all but one participant benefited in either time or accuracy.

Interactions Made Possible by Augmentations. Four participants were not able to interact with the touchscreen interface when no augmentation was provided (P10, P11, P12, and P13). In contrast, augmentations enabled them to find and press targets, requiring 3.0s per button press for familiar and 3.7s for unfamiliar interfaces. Out of their button presses $44 \%$ for familiar and $39 \%$ for unfamiliar interfaces missed the target. Yet, with the help of some augmentations $\mathrm{P} 12$ and $\mathrm{P} 9$ made less than $10 \%$ errors.

The high number of wrong inputs likely results from these four participants not being able to perceive the keyboard due to their low visual acuity. P11 reported "Now that you say it, there is some grey area.". The others noticed the grey area, but could not see letters nor the gap between buttons. Therefore, these participants were not able to use the augmentation to find and then target the correct button, but instead, they had to "blindly" pressed into the center of the augmentation with no means of correcting one's estimated input location against a visual goal. Still, while the number of errors is high, and likely is not acceptable in real-world settings, participants demonstrated performance above chance level and illustrate the potential of the approach. Adaptations, such as adding visual feedback whenever the finger hovers above the correct location, and customization of the augmentations to users' individual visual capabilities, will likely improve the usability. Lastly, as P11 noted, "Using any assistive device needs training, then it will work better".

We have demonstrated that the presented augmentations assist persons with VI when interacting with touchscreen elements. A complete system, which is to work in any environment, additionally requires recognition of touchscreen elements and user inputs as well as knowledge of user intention.

\subsection{Performance Benefits for Individuals Vary across Augmentations (RQ2)}

RQ2 addresses differences between augmentations. We identified and addressed three subquestions: the comparison of global guides and local highlights, of simple crosses and crosses with directional information, and of a stationary cross and a augmentation with motion. To summarize the results, there were no significant differences between any of the groups of augmentations. We attribute this to, often diametrically opposed, differences in individual perception and preference (e.g., "It is hard to hit the middle [for Circle]." - P8 vs. "The circle was the best. The others are hard to orientate [myself]" - P2), which often become apparent from participants' comments.

Local Highlights are Preferred when Target's Location is Known (RQ2.1). We compared global guides against local highlights. While there is no significant difference between these two types of augmentations, local highlights were recommended several times for familiar layouts ("If the circle is in my visual field, it is way easier to hit than the other augmentations" - P9). In other words, local highlights are preferred if the coarse location of the target is known and the user only requires assistance in accurately pressing the button. Global guides, on the other hand, are preferred for unfamiliar interfaces. This is most obvious in situations where the user would have to search for the local highlight, i.e., if the target is outside of the augmentable area of the HoloLens ("Sometimes I have to search. I have to look around to find the circle. That is difficult." - P4; "Because the circle is so small, [for a target outside the augmentable area] the star is helpful, because you see [where] there is something" - P9).

Simple Global Guides Allow for a Wider Range of Users (RQ2.2). We also evaluated the impact of adding directional information to a simple cross augmentation. Most participants favored the visually least complex version SimpleCross out of the global guides, and several reported difficulties to visually parse the more complex variants. ArrowheadCross and especially MovingArrowheadCross were often described as "too much" ("there is just too much going on" - P3) and distracting ("the hooks are giving me more trouble" - P15) and rated as the least preferred. On the other hand, some participants mentioned that often only one line of SimpleCross is 
visible and that directional information has value. Consequently, three of these participants chose Star as their favorite augmentation, mentioning the ease of knowing where to search for the next target based on the directional information.

We conclude that generally a simple representation is sufficient for interfaces that are smaller or not much larger than the augmentable area of the AR glasses, and can assist a wide range of persons with VI. Nevertheless, individual participants can benefit from directional information if the augmentation can be perceived and is not too complex. Further, we assume that the advantage of directional information increases with larger interfaces and decreases with a larger augmentable area in the AR glasses.

Motion does Not Add Additional Benefit (RQ2.3). To evaluate the impact of motion in an augmentation on users' performance, we compared ArrowheadCross against MovingArrowheadCross. Our hypothesis was, that motion provides a benefit. When participants were asked to compare these two augmentations directly, all reported to either note no difference or that the motion distracts and adds clutter, making it harder to find the middle. Therefore, we conclude that motion (in the form of MovingArrowheadCross) adds too much detail to be perceived easily by persons with VI.

\subsection{Augmentations Lower the Perceived Workload (RQ3)}

For our third research question, we evaluated whether the augmentation of an interface lowers the perceived workload. We find that, for familiar interfaces, the perceived workload is reduced and, for unfamiliar interfaces, it is reduced significantly. These findings are confirmed by qualitative feedback describing the baseline condition as difficult and exhausting. There was no difference in the perceived workload between the augmentations. However, interindividual differences might mask such effects, as was suggested by participants' verbal feedback. In sum, our results support the conclusion that augmenting a touchscreen subjectively lowers the participants' effort and the perceived workload of the interaction.

\subsection{Limitations}

Our augmentations proved helpful in assisting persons with VI during the interaction with touchscreen devices. Some users could even correctly press elements on an interface that they could not perceive. We used a setup with an upright touchscreen. For interfaces with greater inclination, which we believe are less common, it might be more challenging for the user to estimate the distance of their finger to the screen and press correctly. However, we are not aware of such effects. Nevertheless, we also noted some challenges. First, there are some technological limitations to overcome. The Microsoft HoloLens has an augmentable field of view of only $30^{\circ}$ horizontally and $17.5^{\circ}$ vertically. At the same time, it is heavy and quite large. Newer HMDs mitigate some of these issues, by providing a larger augmentable area or having a smaller form factor. A wider augmentable field of vision would have allowed P16 to use the system without holding the HoloLens with one hand. Additionally, another user with central vision loss reported during the pilot study that he has to adapt his usual strategies by using eye-movements instead of head-movements to focus the target as otherwise no augmentation was visible. A larger augmentable area may also influence users' performance with the different augmentations as directional information might be less important since the target would not be outside the visible area. Further, improved trackingeven when close to a surface-is required so that users such as P14, who needed close-up inspection of the interface, can use the system. Lastly, the AR glasses need to know the intention of the user, the currently visible layout, and the invisible states of the interface, i.e., the system needs to know the user's goal and the ordered sequence of interface elements one needs to activate to achieve that goal. This is possible using voice commands, crowd-sourcing platforms, and computer vision, as Guo et al. demonstrate with StateLens [8]; however, a versatile context-aware and lightweight system will require newer technology. Regardless, three participants expressed the desire to use the AR glasses even with current hardware and others were excited about the prospect of better AR glasses in the future.

Further challenges result from individual differences between users. We received comments that the augmentations require further adjustments to account for individual capabilities ("I would need the lines a lot thicker" - P11; "I would prefer another color" - P8). Yet, we offered customization only during the design phase and not in the final study to ensure comparability across users. Furthermore, recognizing the augmentations proves challenging for users with very low vision, as discussed in Section 6.3.

\section{CONCLUSION}

We have presented a set of different designs for interface augmentations that improve accuracy and task completion time for VI users in certain scenarios. For individuals with very low visual acuity, augmentation can provide a unique means to access interfaces that are not customizable (e.g., on household appliances) or simply not accessible for users with VI. Being able to interact with a touchscreen without moving ones head close to the device also mitigates stigmatization from bystanders and hygienic issues.

Our results provide guidance for designers and researchers. Users with VI benefit from augmentations when interacting with an interface. Further, visually simple augmentations can be perceived by many persons. We envision that the concept of visual augmentations is applicable for any widget-based interface. However, the effects of visual clutter in more complex or colorful interfaces and of physical interface elements need to be evaluated in future work.

We expect that the usage of AR will be the norm in the long term and technical improvements will mitigate some of the technical limitations. Future devices will provide comfortable everyday use and be adopted across a wide range of users, not only as an assistive device for people with impairments. A system providing such AR assistance for devices in the real world would allow users with VI to interact with machines in a natural and unimpeded manner.

\section{ACKNOWLEDGMENTS}

F. Lang was supported by the German Federal Ministry of Education and Research as part of the project IDeA (grant no. 16SV8102). T. Machulla was supported by the European Union's Horizon2020 Programme under ERCEA grant no. 683008 AMPLIFY. We would also like to thank the reviewers and ACs for their work and valuable feedback. 


\section{REFERENCES}

[1] Felix Bork, Christian Schnelzer, Ulrich Eck, and Nassir Navab. 2018. Towards Efficient Visual Guidance in Limited Field-of-View Head-Mounted Displays. IEEE Transactions on Visualization and Computer Graphics 24, 11 (2018), 2983-2992. https://doi.org/10.1109/TVCG.2018.2868584

[2] Andy Field, Jeremy Miles, and Zoë Field. 2012. Discovering Statistics Using $R$ SAGE Publications Ltd. 978-1-446-20045-2.

[3] Giovanni Fusco, Ender Tekin, Richard E Ladner, and James M Coughlan. 2014 Using computer vision to access appliance displays. In Proceedings of the 16th international ACM SIGACCESS conference on Computers \& accessibility. 281-282.

[4] E.B. Goldstein. 2009. Sensation and Perception. Cengage Learning. https://books. google.de/books?id=2tW91BWeNq4C

[5] Uwe Gruenefeld, Dag Ennenga, Abdallah El Ali, Wilko Heuten, and Susanne Boll. 2017. EyeSee360: Designing a Visualization Technique for out-of-View Objects in Head-Mounted Augmented Reality. In Proceedings of the 5th Symposium on Spatial User Interaction (Brighton, United Kingdom) (SUI '17). Association for Computing Machinery, New York, NY, USA, 109-118. https://doi.org/10.1145/ 3131277.3132175

[6] Anhong Guo, Xiang Chen, Haoran Qi, Samuel White, Suman Ghosh, Chieko Asakawa, and Jeffrey Bigham. 2016. VizLens: A Robust and Interactive Screen Reader for Interfaces in the Real World. 651-664. https://doi.org/10.1145/2984511. 2984518

[7] Anhong Guo, Jeeeun Kim, Xiang 'Anthony' Chen, Tom Yeh, Scott E. Hudson, Jennifer Mankoff, and Jeffrey P. Bigham. 2017. Facade: Auto-Generating Tactile Interfaces to Appliances. In Proceedings of the 2017 CHI Conference on Human Factors in Computing Systems (Denver, Colorado, USA) (CHI '17). Association for Computing Machinery, New York, NY, USA, 5826-5838. https://doi.org/10.1145/ 3025453.3025845

[8] Anhong Guo, Junhan Kong, Michael Rivera, Frank F. Xu, and Jeffrey P. Bigham. 2019. StateLens: A Reverse Engineering Solution for Making Existing Dynamic Touchscreens Accessible. In Proceedings of the 32nd Annual ACM Symposium on User Interface Software and Technology (New Orleans, LA, USA) (UIST '19). Association for Computing Machinery, New York, NY, USA, 371-385. https: //doi.org/10.1145/3332165.3347873

[9] Sandra Hart. 2006. Nasa-task load index (Nasa-TLX); 20 years later. Proceedings of the Human Factors and Ergonomics Society Annual Meeting 50. https://doi.org/ $10.1177 / 154193120605000909$

[10] Sandra G Hart and Lowell E Staveland. 1988. Development of NASA-TLX (Task Load Index): Results of empirical and theoretical research. Human mental workload 1, 3 (1988), 139-183.

[11] Jonathan Huang, Max Kinateder, Matt Dunn, Wojciech Jarosz, Xing-Dong Yang, and Emily Cooper. 2019. An augmented reality sign-reading assistant for users with reduced vision. PLOS ONE 14 (01 2019), e0210630. https://doi.org/10.1371/ journal.pone. 0210630

[12] Yvonne Jansen, Thorsten Karrer, and Jan Borchers. 2010. MudPad: Tactile Feedback and Haptic Texture Overlay for Touch Surfaces. In ACM International Conference on Interactive Tabletops and Surfaces (Saarbrücken, Germany) (ITS '10). Association for Computing Machinery, New York, NY, USA, 11-14 https://doi.org/10.1145/1936652.1936655

[13] Albouys Perrois Jérémy, Jeremy Laviole, Carine Briant, and Anke Brock. 2018. Towards a Multisensory Augmented Reality Map for Blind and Low Vision People: a Participatory Design Approach. 1-14. https://doi.org/10.1145/3173574.3174203

[14] Shaun Kane, Meredith Morris, and Jacob Wobbrock. 2013. Touchplates: Low-Cost Tactile Overlays for Visually Impaired Touch Screen Users. Proceedings of the 15th International ACM SIGACCESS Conference on Computers and Accessibility, ASSETS 2013. https://doi.org/10.1145/2513383.2513442

[15] Shaun K. Kane, Chandrika Jayant, Jacob O. Wobbrock, and Richard E. Ladner. 2009. Freedom to Roam: A Study of Mobile Device Adoption and Accessibility for People with Visual and Motor Disabilities. In Proceedings of the 11th International ACM SIGACCESS Conference on Computers and Accessibility (Pittsburgh, Pennsylvania, USA) (Assets '09). Association for Computing Machinery, New York, NY, USA, 115-122. https://doi.org/10.1145/1639642.1639663

[16] Junhan Kong, Anhong Guo, and Jeffrey P. Bigham. 2019. Supporting Older Adults in Using Complex User Interfaces with Augmented Reality. In The 21st International ACM SIGACCESS Conference on Computers and Accessibility (Pittsburgh, PA, USA) (ASSETS '19). Association for Computing Machinery, New York, NY, USA, 661-663. https://doi.org/10.1145/3308561.3354593

[17] Koolertron. 2021. http://www.koolertron.com/electronics-digital-magnifier-c106_108.html

[18] Florian Lang, Albrecht Schmidt, and Tonja Machulla. 2020. Augmented Reality for People with Low Vision: Symbolic and Alphanumeric Representation of Information. In Computers Helping People with Special Needs, Klaus Miesenberger Roberto Manduchi, Mario Covarrubias Rodriguez, and Petr Peňáz (Eds.). Springer International Publishing, Cham, 146-156.

[19] Anabel Martín-González. 2011. Advanced imaging in head-mounted displays for patients with age-related macular degeneration. Ph.D. Dissertation. Technical University Munich. http://nbn-resolving.de/urn:nbn:de:bvb:91-diss-20110928-
1079926-1-1

[20] David Mcgookin, Stephen Brewster, and WeiWei Jiang. 2008. Investigating Touchscreen Accessibility for People with Visual Impairments. ACM International Conference Proceeding Series 358. https://doi.org/10.1145/1463160.1463193

[21] Suzanne Mckee and Ken Nakayama. 1984. The Detection of Motion in the Peripheral Visual Field. Vision research 24 (02 1984), 25-32. https://doi.org/10. 1016/0042-6989(84)90140-8

[22] NuEyes. 2021. https://nueyes.com/

[23] OrCam. 2021. https://www.orcam.com

[24] D. T. V. Pawluk, R. J. Adams, and R. Kitada. 2015. Designing Haptic Assistive Technology for Individuals Who Are Blind or Visually Impaired. IEEE Transactions on Haptics 8, 3 (2015), 258-278. https://doi.org/10.1109/TOH.2015.2471300

[25] Patrick Renner and Thies Pfeiffer. 2017. Attention guiding techniques using peripheral vision and eye tracking for feedback in augmented-reality-based assistance systems. 186-194. https://doi.org/10.1109/3DUI.2017.7893338

[26] Teresa Siu and Valeria Herskovic. 2013. SidebARs: Improving Awareness of off-Screen Elements in Mobile Augmented Reality. In Proceedings of the 2013 Chilean Conference on Human - Computer Interaction (Temuco, Chile) (ChileCHI '13). Association for Computing Machinery, New York, NY, USA, 36-41. https: //doi.org/10.1145/2535597.2535608

[27] Lee Stearns, Victor DeSouza, Jessica Yin, Leah Findlater, and Jon Froehlich. 2017. Augmented Reality Magnification for Low Vision Users with the Microsoft Hololens and a Finger-Worn Camera. 361-362. https://doi.org/10.1145/ 3132525.3134812

[28] VPIXX Technologies. 2021. https://vpixx.com/products/touch-panel/

[29] Ingrid U Scott, William Feuer, and Julie Jacko. 2002. Impact of graphical user interface screen features on computer task accuracy and speed in a cohort of patients with age-related macular degeneration. American journal of ophthalmology 134 (12 2002), 857-62. https://doi.org/10.1016/S0002-9394(02)01795-6

[30] Ingrid U Scott, William Feuer, and Julie Jacko. 2002. Impact of Visual Function on Computer Task Accuracy and Reaction Time in A Cohort of Patients With Age-Related Macular Degeneration. American journal of ophthalmology 133 (03 2002), 350-7. https://doi.org/10.1016/S0002-9394(01)01406-4

[31] Xiaoyi Zhang, Tracy Tran, Yuqian Sun, Ian Culhane, Shobhit Jain, James Fogarty, and Jennifer Mankoff. 2018. Interactiles: 3D Printed Tactile Interfaces to Enhance Mobile Touchscreen Accessibility. In Proceedings of the 20th International ACM SIGACCESS Conference on Computers and Accessibility (Galway, Ireland) (ASSETS '18). Association for Computing Machinery, New York, NY, USA, 131-142. https: //doi.org/10.1145/3234695.3236349

[32] Yuhang Zhao, Edward Cutrell, Christian Holz, Meredith Ringel Morris, Eyal Ofek, and Andrew D. Wilson. 2019. SeeingVR: A Set of Tools to Make Virtual Reality More Accessible to People with Low Vision. 1-14. https://doi.org/10. 1145/3290605.3300341

[33] Yuhang Zhao, Elizabeth Kupferstein, Brenda Veronica Castro, Steven Feiner, and Shiri Azenkot. 2019. Designing AR Visualizations to Facilitate Stair Navigation for People with Low Vision. In Proceedings of the 32nd Annual ACM Symposium on User Interface Software and Technology (New Orleans, LA, USA) (UIST '19). Association for Computing Machinery, New York, NY, USA, 387-402. https: //doi.org/10.1145/3332165.3347906

[34] Yuhang Zhao, Sarit Szpiro, and Shiri Azenkot. 2015. ForeSee: A Customizable Head-Mounted Vision Enhancement System for People with Low Vision. 239-249. https://doi.org/10.1145/2700648.2809865

[35] Yuhang Zhao, Sarit Szpiro, Jonathan Knighten, and Shiri Azenkot. 2016. CueSee: exploring visual cues for people with low vision to facilitate a visual search task. 73-84. https://doi.org/10.1145/2971648.2971730

[36] ZoomText. 2021. https://www.zoomtext.com/ 